\title{
Teaching Physical Examination to Medical Students on Inpatient Medicine Teams: A Prospective, Mixed-Methods Descriptive Study
}

\author{
Paul A. Bergl, MD $1, \star$, Allison C. Taylor, BS², Jennifer Klumb, MS², Kerrie Quirk, MA², \\ Martin D. Muntz, MD2, Kathlyn E. Fletcher, MD, MA²
}

${ }^{1}$ Medical College of Wisconsin Affiliated Hospitals, Milwaukee, Wisconsin. At the time of this study, Dr. Bergl was with the Division of General Internal Medicine, Medical College of Wisconsin, Milwaukee, Wisconsin. ${ }^{2}$ Medical College of Wisconsin, Milwaukee, Wisconsin.

Physical examination (PE) is a core clinical competency, and the internal medicine clerkship is a premiere venue for students to develop PE skills. However, clinical rotations often lack opportunities for real-time instruction. We sought to measure the frequency, content, and factors affecting PE instruction during the internal medicine clerkship. We conducted a prospective mixed-methods study at a single academic center. Data were gathered by a student researcher who directly observed inpatient teams over 3 months. We quantified the frequency of PE teaching activities and analyzed daily written observations using qualitative content analysis. PE was most frequently discussed during bedside rounds and least often during workroom rounds. Direct observation of students' examinations rarely occurred. Multiple factors in the learning environment were posited to affect PE instruction. In brief, we found that residents and attending physicians who are part of internal medicine teaching services do not routinely emphasize PE instruction. Journal of Hospital Medicine 2018;13:399-402. @ 2018 Society of Hospital Medicine

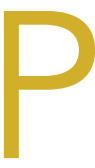

hysical examination (PE) is a core clinical skill in undergraduate medical education. ${ }^{1}$ Although the optimal approach to teaching clinical skills is debated, robust preclinical curricula should generally be followed by iterative skill development during clinical rotations. ${ }^{2,3}$

The internal medicine rotation represents a critical time to enhance PE skills. Diagnostic decision making and PE are highly prioritized competencies for the internal medicine clerkship, ${ }^{4}$ and students will likely utilize many core examination skills ${ }^{1,2}$ during this time. Bedside teaching of PE during the internal medicine service also provides an opportunity for students to receive feedback based on direct observation, ${ }^{5}$ a sine qua non of competency-based assessment.

Unfortunately, current internal medicine training environments limit opportunities for workplace-based instruction in PE. Recent studies suggest diminishing time spent on bedside patient care and teaching, with computer-based "indirect patient care" dominating much of the clinical workday of internal medicine services. ${ }^{6-8}$ However, the literature does not delineate how often medical students are enhancing their PE skills during clinical rotations or describe how the educational environment may influence PE teaching.

\footnotetext{
*Address for correspondence: Paul Bergl, MD, Medical College of Wisconsin, 9200 W. Wisconsin Ave., $4^{\text {th }}$ floor, Specialty Clinics, Milwaukee, WI 53226; Telephone: 414-955-7040; Fax: 414-955-0175; E-mail: pbergl@mcw.edu

Additional Supporting Information may be found in the online version of this article.
}

Received: November 17, 2017; Revised: February 8, 2018; Accepted: February 23,2018

๑ 2018 Society of Hospital Medicine DOI 10.12788/jhm.2972
We aimed to describe the content and context of PE instruction during the internal medicine clerkship workflow. Specifically, we sought to explore what strategies physician team members used to teach PE to students. We also sought to describe factors in the inpatient learning environment that might explain why physical examination (PE) instruction occurs infrequently.

\section{METHODS}

We conducted a prospective mixed-methods study using time motion analysis, checklists on clinical teaching, and daily open-ended observations written by a trained observer from June through August 2015 at a single academic medical center. Subjects were recruited from internal medicine teaching teams and were allowed to opt out. Teaching teams had 2 formats: (1) traditional team with an attending physician (hospitalist or general internist), a senior resident, 2 interns, a fourth-year medical student, and 2 third-year students or (2) hospitalist team in which a third-year student works directly with a hospitalist and advanced practitioner. The proposal was submitted to the Medical College of Wisconsin Institutional Review Board and deemed exempt from further review.

All observations were carried out by a single investigator (A.T.), who was a second-year medical student at the time. To train this observer and to pilot the data collection instruments, our lead investigator (P.B.) directly supervised our observer on 4 separate occasions, totaling over 12 hours of mentored co-observation. Immediately after each training session, both investigators (A.T. and P.B.) debriefed to compare notes, to review checklists on recorded observations, and to discuss areas of uncertainty. During the training period, formal metrics of agreement (eg, kappa coefficients) were not gathered, as data 
collection instruments were still being refined.

Observation periods were centered on third-year medical students and their interactions with patients and members of the teaching team. Observed activities included pre-rounding, teaching rounds with the attending physician, and new patient admissions during call days. Observations generally occurred between the hours of $7 \mathrm{AM}$ and $6 \mathrm{PM}$, and we limited periods of observation to 3 consecutive hours to minimize observer fatigue. Observation periods were selected to maximize the number of subjects and teams observed, to adequately capture pre-rounding and new admissions activities, and to account for variations in rounding styles throughout the call cycle. Teams were excluded if a member of the study team was an attending physician on the clinical team or if any member of the patient care team had opted out of the study.

Data were collected on paper checklists that included idealized bedside teaching activities around PE. Teaching activities were identified through a review of relevant literature, ${ }^{9,10}$ and were further informed by our senior investigator's own experience with faculty development in this area ${ }^{11}$ and team members' attendance at bedside teaching workshops. At the end of each day, our observer also wrote brief observations that summarized factors affecting bedside teaching of PE. Checklist data were transferred to an Excel file (Microsoft), and written observations were imported into NVivo 10 (ORS International, Melbourne, Australia) for coding and analysis.

Checklist data were analyzed using simple descriptive statistics. We compared time spent on various types of rounding using ANOVA, and we used a Student two-tailed t-test to compare the amount of time students spent examining patients on pre-rounds versus new admissions. To ascertain differences in the frequency of PE teaching activities by location, we used chi-squared tests. Statistical analysis was performed using embedded statistics functions in Microsoft Excel. A P value of $<.05$ was used as the cut-off for significance.

We analyzed the written observations using conventional qualitative content analysis. Two investigators (A.T. and P.B.) reviewed the written comments and used open coding to devise a preliminary inductive coding scheme. Codes were refined iteratively, and a schema of categories and nodes was outlined in a codebook that was periodically reviewed by the entire research team. The coding investigators met regularly to ensure consistency in coding, and a third team member remained available to reconcile significant disagreements in code definitions.

\section{RESULTS}

Eighty-one subjects participated in the study: 21 were attending physicians, 12 residents, 21 interns, 11 senior medical students, and 26 junior medical students. We observed 16 distinct inpatient teaching teams and 329 unique patient-related events (discussions and/or patient-clinician encounters), with most events being observed during attending rounds (269/329, or $82 \%)$. There were 123 encounters at the bedside, averaging 7 minutes; 43 encounters occurred in the hallway, averaging 8 minutes each; and 163 encounters occurred in a workroom and averaged 7 minutes per patient discussion. We also observed 28 student-patient encounters during pre-round activities and 30 student-patient encounters during new admissions.

\section{Teaching and Direct Observation}

During attending rounds at the bedside, the attending physician examined the patient 82 times out of 123 patient encounters (67\%). Teaching activities during these PEs were mostly limited to the attending physician or senior resident noting findings (37 instances out of 82 examinations, or 45\%). Rarely did the teacher ask students to re-examine the patient before revealing relevant findings ( 5 instances out of 82 examinations, or $6 \%$ ), and only during $15 \%$ of bedside examinations did the attending physician directly observe students performing a portion of the PE. As demonstrated in Table 1, discussions at the bedside were more likely to reference the PE $(P<.001$, chi-squared) and more often resulted in specific plans to verify physical findings $(P<.001$, chi-squared) compared with patient-related discussions in other settings. The location of rounding activities, however, did not affect how often teams incorporated $\mathrm{PE}$ into clinical decision-making $(P=.82)$.

During 28 pre-rounding encounters, students usually examined the patient (26 out of 28 instances, 93\%) but were observed only 4 times doing so (out of 26 instances, or $15 \%$ ). During 30 new patient admissions, students examined 27 patients (90\%) and had their PE observed 6 times (out of 27 instances, or $22 \%$ ). There were no significant differences in frequency of these activities $(P>.05$, chi-squared) between prerounds or new admissions.

\section{Observations on Teaching Strategies}

In the written observations, we categorized various methods being used to teach PE. Bedside teaching of PE most often involved teachers simply describing or discussing physical findings (42 mentions in observations) or verifying a student's reported findings ( 15 mentions). Teachers were also observed to use bedside teaching to contextualize findings (13 mentions), such as relating the quality of bowel sounds to the patient's constipation or to discuss expected pupillary light reflexes in a neurologically intact patient. Less commonly, attending physicians narrated steps in their PE technique (9 mentions). Students were infrequently encouraged to practice a specific PE skill again (7 mentions) or allowed to re-examine and reconsider their initial interpretations (5 mentions).

Our written observations also identified factors that may impact clinical instruction of PE as shown in Table 2. In the learning environment, physical space, place, and timing of teaching moments all impacted PE teaching on the wards. Clinical workload and a focus on efficiency appeared to diminish the quality of PE instruction, such as by limiting the number of participants or by leading teams to conduct "sit-down rounds" in workrooms.

\section{DISCUSSION}

This observational study of clinical teaching on internal medicine teaching services demonstrates that PE teaching is most likely to occur during bedside rounding. However, even in bed- 
TABLE 1. Clinical and Teaching Activities on Attending Rounds

\begin{tabular}{|c|c|c|c|c|}
\hline & \multirow[b]{2}{*}{ Bedside } & \multicolumn{2}{|c|}{ Other Settings } & \multirow[b]{2}{*}{ P Vvalues (Bedside vs. Other Settings) } \\
\hline & & Hallway & Workroom & \\
\hline Total encounters & 123 & 43 & 163 & \\
\hline Total time spent rounding per patient & 7 minutes & 8 minutes & 7 minutes & Not significant \\
\hline Mentioned or performed PE & $82(66 \%)$ & $24(56 \%)$ & $72(44 \%)$ & $P=.0008$ \\
\hline \multicolumn{5}{|l|}{ When PE was mentioned or performed, team... } \\
\hline Noted important PE findings to verify & $37 / 82(45 \%)$ & $3 / 24(13 \%)$ & $5 / 72(7 \%)$ & $\mathrm{P}<.00001$ \\
\hline Incorporated PE into patient care and clinical decision-making & $16 / 82(20 \%)$ & $5 / 24(21 \%)$ & $17 / 72(24 \%)$ & Not significant \\
\hline
\end{tabular}

${ }^{a} P$ values calculated by chi-squared tests. NOTE: Abbreviation: PE, physical examination.

TABLE 2. Key Factors in the Clinical Environment That May Have Influenced PE Instruction

\begin{tabular}{|c|c|}
\hline Variable & Relationship to PE Instruction with Salient Example \\
\hline \multirow[t]{2}{*}{ Physical space and location } & The bedside appears to be the ideal location for PE teaching. \\
\hline & Example: Direct observation with real-time feedback by the attending was only seen in this setting. \\
\hline \multirow[t]{2}{*}{ Number of participants } & Bedside PE instruction may be more common with one-on-one interactions. \\
\hline & $\begin{array}{l}\text { Example: A junior student noted that being on hospitalist service provided more opportunities for patient care responsibilities and a more intimate dynamic } \\
\text { for bedside teaching. }\end{array}$ \\
\hline \multirow[t]{2}{*}{ Timing } & Teaching about PE may be more effective immediately prior to the actual patient encounter. \\
\hline & Example: Attending reviewed the pathophysiology of heart failure in the hallway immediately before visiting the patient. \\
\hline \multirow[t]{2}{*}{ Patient participation } & There is a tendency to dismiss physical findings when discussed away from the patient. \\
\hline & Example: Teams appeared to brush over "disembodied" findings during post-call conference room presentations. \\
\hline \multirow[t]{2}{*}{ Clinical workload } & Clinical efficiency by house officers is prioritized over directly observing students. \\
\hline & Example: An intern typed a new admission note and put in orders while the junior student interviewed and examined the patient. \\
\hline \multirow[t]{2}{*}{ Access to technology } & Appropriately used technology can potentially enhance PE teaching. \\
\hline & Example: A fourth-year student showed the team a picture of a sacral wound that he took on his smartphone. \\
\hline
\end{tabular}

NOTE: Abbreviation: PE, physical examination.

side encounters, most PE instruction is limited to physician team members pointing out significant findings. Although physical findings were mentioned for the majority of patients seen on rounds, attending physicians infrequently verified students' or residents' findings, demonstrated technique, or incorporated PE into clinical decision making. We witnessed an alarming dearth of direct observation of students and almost no real-time feedback in performing and teaching PE. Thus, students rarely had opportunities to engage in higher-order learning activities related to $\mathrm{PE}$ on the internal medicine rotation.

We posit that the learning environment influenced PE instruction on the internal medicine rotation. To optimize inpatient teaching of $\mathrm{PE}$, attending physicians need to consider the factors we identified in Table 2. Such teaching may be effective with a more limited number of participants and without distraction from technology. Time constraints are one of the major perceived barriers to bedside teaching of $\mathrm{PE}$, and our data support this concern, as teams spent an average of only 7 minutes on each bedside encounter. However, many of the strategies observed to be used in real-time PE instruction, such as validating the learners' findings or examining patients as a team, naturally fit into clinical routines and generally do not require extra thought or preparation.

One of the key strengths of our study is the use of direct observation of students and their teachers. This study is unique in its exclusive focus on PE and its description of factors affecting $P E$ teaching activities on an internal medicine service. This observational, descriptive study also has obvious limitations. The study was conducted at a single institution during a limited time period. Moreover, the study period June through August, which was chosen based on our observer's availability, includes the transition to a new academic year (July 1, 2015) when medical students and residents were becoming acclimated to their new roles. Additionally, the data were collected by a single re- 
searcher, and observer bias may affect the results of qualitative analysis of journal entries.

In conclusion, this study highlights the infrequency of applied PE skills in the daily clinical and educational workflow of internal medicine teaching teams. These findings may reflect a more widespread problem in clinical education, and replication of our findings at other teaching centers could galvanize faculty development around bedside PE teaching.

Disclosures: Dr. Bergl has nothing to disclose. Ms. Taylor reports grant support from the Cohen Endowment for Medical Student Research at the Medical College of Wisconsin during the conduct of the study. Mrs. Klumb, Ms. Quirk, Dr. Muntz, and Dr. Fletcher have nothing to disclose.

Funding: This work was funded in part by the Cohen Endowment for Medical Student Research at the Medical College of Wisconsin.

\section{References}

1. Corbett $E$, Berkow R, Bernstein $L$, et al on behalf of the AAMC Task Force on the Preclerkship Clinical Skills Education of Medical Students. Recommendations for clinical skills curricula for undergraduate medical education. Achieving excellence in basic clinical method through clinical skills education: The medical school clinical skills curriculum. Association of American Medical Colleges; 2008. https://www.aamc.org/download/130608/data/clin- icalskills_oct09.qxd.pdf.pdf. Accessed July 12, 2017.

2. Gowda D, Blatt B, Fink MJ, Kosowicz LY, Baecker A, Silvestri RC. A core physical exam for medical students: Results of a national survey. Acad Med. 2014;89(3):436-442.

3. Uchida T, Farnan JM, Schwartz JE, Heiman HL. Teaching the physical examination: A longitudinal strategy for tomorrow's physicians. Acad Med. 2014;89(3):373-375.

4. Fazio S, De Fer T, Goroll A. Core Medicine Clerkship Curriculum Guide: A resource for teachers and learners. Clerkship Directors in Internal Medicine and Society of General Internal Medicine; 2006. http://www.im.org/d/ do/2285/. Accessed July 12, 2017.

5. Gonzalo J, Heist B, Duffy B, et al. Content and timing of feedback and reflection: A multi-center qualitative study of experienced bedside teachers. BMC Med Educ. 2014;(14):212. doi: 10.1186/1472-6920-14-212.

6. Stickrath C, Noble M, Prochazka A, et al. Attending rounds in the current era: What is and is not happening. JAMA Intern Med. 2013;173(12):1084-1089.

7. Block L, Habicht R, Wu AW, et al. In the wake of the 2003 and 2011 duty hours regulations, how do internal medicine interns spend their time? J Gen Intern Med. 2013;28(8):1042-1047.

8. Wenger N, Méan M, Castioni J, Marques-Vidal P, Waeber G, Garnier A. Allocation of internal medicine resident time in a Swiss Hospital: A time and motion study of day and evening shifts. Ann Intern Med. 2017;166(8):579-586.

9. Ramani S. Twelve tips for excellent physical examination teaching. Med Teach. 2008;30(9-10):851-856.

10. Gonzalo JD, Heist BS, Duffy BL, et al. The art of bedside rounds: A multi-center qualitative study of strategies used by experienced bedside teachers. $J$ Gen Intern Med. 2013;28(3):412-420.

11. Janicik RW, Fletcher KE. Teaching at the bedside: A new model. Med Teach. 2003;25(2):127-130. 